\title{
Research on Geophone Array Method and Forward and Inversion Algo- rithm in Micro-seismic Positioning and Monitoring System
}

\author{
Faquan Zhang*, Xiaozhe Yang, Pengfei Wang, Jincai Ye and Guofu Wang
}

School of Information and Communication, Guilin University of Electronic Technology, Guilin 541004, China

\begin{abstract}
In order to make the seismic positioning and monitoring system work more effectively, this paper designs four kinds of geophone array methods. By establishing complex geological model and using micro-grid ray tracing, the process that the signal starts from the source location to the seismic geophone arrays is simulated in forward algorithm. In condition of random disturbance, the source location is retrieved by means of inversion algorithm fulfilled by neural network algorithm and genetic algorithm. Finally, on the basis of the analysis of the result and comparison with the true location, the characteristics of every geophone array method are presented. The paper also discusses proper number of geophones in the every array method. Results show that the most accurate, reasonable and effective array method can be selected and the forward and inversion algorithm is also effective.
\end{abstract}

Keywords: Genetic algorithm, geophone array, seismic positioning and monitoring, source location.

\section{INTRODUCTION}

Illegal coal mining is still hard to be found; the supervision department urgently needs a set of positioning and monitoring equipment which possesses high detection precision and short testing periodic time to detect the underground situation. Seismic positioning and monitoring is now the most accurate, timely as well as the most informative method, which is used in mining area to detect illegal coal mining [1-3]. It is also a geophysical technique that is based on acoustic emission and seismology. Hence it can reflect the effect of seismic events in stratum and some activities in the underground. One of the main tasks of the seismic positioning and monitoring system is to locate where the shock is [46]. Especially, the geophone array plays a key role in the system as it determines whether the system could work effectively. However, there are few references regarding how the array affects the precision and the reliability of the system and optimization of the geophone array [7, 8]. Therefore, an urgent research is needed on the subject [9-12]. Wang jian et al. researched optimal design of geophone network in seismic monitoring. But they did not take into consideration the effect of various geophone arrays on computational efficiency and accuracy of the source location, especially in complicated formation along with the condition of noise disturbance. Wu Jianxing researched on geophone network arrangement in seismic monitoring system. But geophones were only arranged in order in the tunnel of coal mine and the proper quantity of geophones was also not provided in detail. Hu Guangjun et al. researched on arrangement of geophones in seismic monitoring and determined the style of cross type to 8 transducers. However, they did not research on the rationality of its style and its efficiency. This paper

*Address correspondence to this author at the School of Information and Communication, Guilin University of Electronic Technology, Guilin 541004, China; Tel: +86-152-9600-6766; E-mail: zhangfq@guet.edu.cn uses GA-BP and micro-grid ray tracing forward algorithm to study the geophone array in order to make seismic positioning and monitoring system more scientific.

\section{FORWARD AND INVERSION ALGORITHM AND ARRAY METHOD DESIGN}

\subsection{Inversion Algorithm of the Source Location Based on GA-BP}

The basic principle of the source location algorithm is to estimate the target location and the time when it provides the signals based on the ones which come from the known observation points. When a certain number of geophones are arranged in a certain way around the source location, they make up the geophone array and the geophones pick up the signals in the presence of an earthquake. By collecting synchronous data from different observation points, the time when each geophone receives the signals can be calculated. Substituting the coordinates of each geophone and the time into the equation, the spatial parameters and the time parameters are obtained. In fact, location of the source is also a process related to the problem of nonlinear equations. This paper solves these problems through GA-BP and by using the nonlinear fitting capacity of $\mathrm{BP}$ and the nonlinear findbest ability of GA in order to find out the source location.

The specific procedures of mixed GA-BP algorithm are as follows:

(1) According to the features of optimization problem, the input, hidden and output layers of neural network can be produced. Moreover, numerous link weights and thresholds can be generated randomly and can be taken as the initial population.

(2) Using data standardization method, neural network with normalization learning samples is provided including input samples and output samples. 
(3) Calculating the error $\mathrm{E}$ of $\mathrm{N}$ groups of initial populations, it is judged whether $\mathrm{E}$ is less than or equal to the preset thresholds. If it is, then the iteration is stopped. If not, the gradient error is sought and the weights and thresholds are corrected.

(4) For determining the coding mode of weights and thresholds, fitness function is chosen as shown in equation (1) to calculate the individual fitness value.

$$
F(x)=\left\{\begin{array}{c}
f(x)-F(x) \text { if } g_{j}(x) \geq 0 \forall j=1,2, \cdots, N \\
f_{\max }+\sum_{j=1}^{m}\left(g_{j}(x)\right)-\overline{F(x)} \quad \text { other }
\end{array}\right.
$$

In this formula, $f(x)$ is individual object function value, $f_{\max }$ is maximum individual fitness, $F(x)$ is average adaption of previous generation, and $\mathrm{g}_{j}(x)$ is output of node $\mathrm{j}$.

(5) After a series of GA calculations, a new population arises. On the basis of repeated calculations within the preset terminating numbers of iteration, it is made sure that every breeding individual is checked. Thus, the population efficiency is improved.

(6) The process is then preceded onto step (3).

\subsection{Forward Simulation Algorithm Based on Micro-grid Ray Tracing}

The best motion track for the seismic wave when it travels in the medium can be found out by Ray Tracing Theory [6]. The theory shows the field energy distribution of the seismic wave, and helps in calculating the coefficient of reflection and projection, simulating the phase and amplitude of many kinds of wave fields. Therefore, it comprises the theoretical basis for tomography inversion. According to the characteristics of seismic signal, wave equation is hard to simulate the real environment whereas the ray tracing theory takes the advantage of making full use of the real microseisms to record information. Besides, calculating speed of the ray tracing method is quicker than wave equation. Therefore, ray tracing theory is used to complete forward simulation calculation.

The complicated geological model is established to accomplish the aim of forward simulation of seismic by combining the trial ray method with micro-grid method in ray tracing method. The real geological condition is far more complicated, because it has complex interface and very rapid velocity gradient changes. In such a model, by discretizing the complex geological model into small grids, the problem can be solved by the micro-grid ray tracing method.

After conducting analysis on the features of seismic monitoring work, the complex geological model is set up through the following steps.

(1) To establish initial model and input of the coordinates of the geophone array and source location, the geological model is meshed in equal space, setting up the range of firing angle while giving consideration to the features of the model.

(2) To start from the grid of the source location, each grid endpoint is regarded as an exit angle, and each grid is traced to its exit angle to obtain the position of intersection.
(3) To add small disturbance to the incident edge of the grid, the next grid is obtained. The step is repeated till the monitoring well intersects the ray, and when they are in the same grid, the procedure turns to the following steps. Otherwise, the original grid area is reset and the procedure is turned to step (3).

(4) To judge the relation of the intersection position of monitoring point and monitoring well, and if it corresponds with the seismic precision, step (5) is followed. If not, after resetting the grid area by dichotomy, step (2) is followed.

(5) To record the ray path and exit angle, the ray travel time can be calculated and the output is then noted.

Suppose when the seismic wave spreads in the medium discrete unit, if the position, slowness and slowness gradient of the discrete unit as well as the position and direction of the ray incident point are already known, the problem can be solved by calculating the position and direction of the ray exit point. The trait of this method is that elements can be changed slightly to cater to the needs of incidence point of the ray. Compared to the traditional method which makes the ray adapt to the grids, it is closer to the objective ray path tracing. It can reduce the iteration times, improve the computational accuracy and speed and decrease the error. The mathematical description is as follows.

In heterogeneous medium model, suppose in medium discrete unit, the gradient of the wave velocity is micro constant, the Eikonal Equation is:

$\frac{d}{d l}\left[s(r) \frac{d r}{d l}\right]=\nabla_{r} s(r)$

In the equation, $s(r)$ and $l$ represent respectively the slowness and arc length, the ray integral form can be expressed as follows:

$r(l)=r_{0}+n_{0} \int_{0}^{1} \frac{s\left(r_{0}\right)}{s\left(r^{\prime}\right)} d l_{0}^{\prime}+\int_{0}^{1} \frac{1}{s\left(r^{\prime}\right)} \int_{0}^{1} \nabla_{r} s\left(r^{\prime \prime}\right) d l^{\prime \prime} d l^{\prime}$

where $r_{0}$ and $n_{0}$ represent the position and direction of $l$ when it is zero. If the medium gradient is constant $G$, the equation is:

$G=\frac{\nabla_{r} s(r)}{s(r)}$

To substitute equation (4) into equation (3), equation (3) can be integrated by using approximation. In $(\mathrm{x}, \mathrm{z})$ coordinates, the component and travel time of $r_{i}$ and $n_{i}$ are expressed as follows:

$$
\left\{\begin{array}{c}
r_{i}(l)=r_{0 i}+n_{0 i}\left[l+\frac{l^{2}}{2}\left(u_{i}-k\right)+\frac{l^{3}}{6}\left(3 k^{2}-G^{2}-2 k u_{i}\right)\right] \\
n_{i}(l)=n_{0 i}\left[l+l\left(u_{i}-k\right)+\frac{l^{3}}{2}\left(3 k^{2}-G^{2}-2 k u_{i}\right)\right] \\
t(l)=l S_{0}\left[l+\frac{1}{2} l+\frac{l^{2}}{6}\left(G^{2}-k^{2}\right)\right]
\end{array}\right.
$$


where $n_{i}(l)$ is ray directional function, $r_{i}(l)$ is ray position vector function, $t(l)$ is travel time function.

\subsection{Geophone Array}

At present, there are still some problems to be solved in the application of seismic monitoring technique, either in theory or in technology such as the feasibility of geophone arrangement [13]. Some array schemes are designed, as seen in Fig. (1). In order to improve location precision of the source and determine reasonable geophone arrangement style and appropriate number of geophone, MATLAB programming tool is used to retrieve the location of the source by inversion. The software is used to calculate the positioning error of the source point and draw out the coordinate error graph. When the geophones are arranged in cruciform shape, M-word shape, square-like shape and octagonal shape, their positioning error graphs are compared and their location precision is analyzed, thus a more reasonable disposal and assessment is provided.
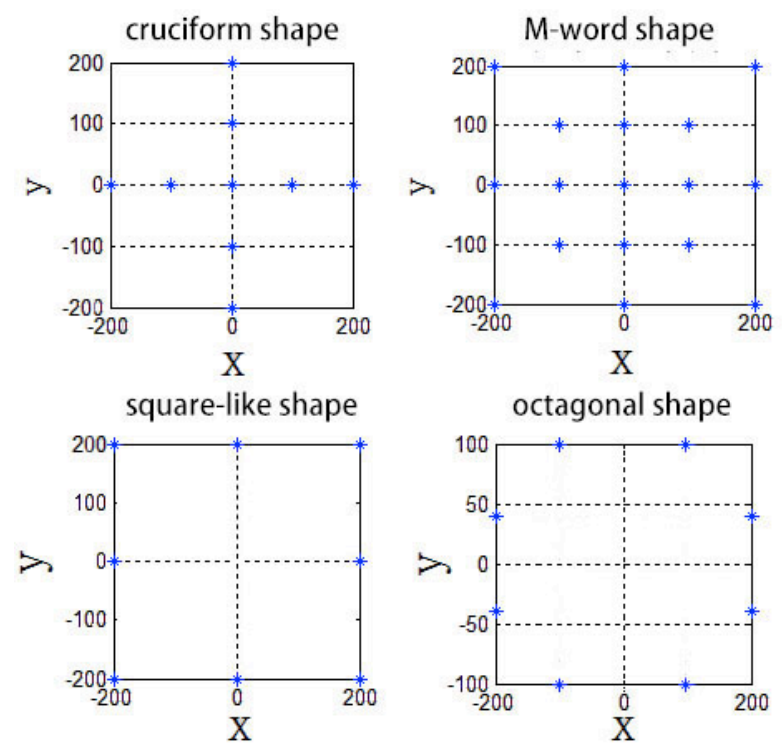

Fig. (1). Four kinds of geophone array diagram.

\section{NUMERICAL SIMULATION EXPERIMENT AND ANALYSIS RESULTS}

\subsection{Establish Mathematical Model of Complex Medium}

Suppose $R_{j}$ and $S_{i}$ respectively represent the geophone and the seismic source, $S_{i}(x, y, z)$ represents the real space coordinates of the ith source. $R_{j}(x, y, z)$ represents the space coordinates of jth geophone monitoring point. In seismic monitoring method, borehole joint monitoring is chosen to set up a complex geological model which is a cube of $1 \mathrm{~km}$ $\times 1 \mathrm{~km} \times 1 \mathrm{~km}$, as shown in Fig. (2). This 3 -D model is made up of two uniform mediums and one heterogeneous medium. In this model, the first interface is tilted interface $R_{1}$, the second is horizontal interface $R_{2}: \mathrm{z}=-800 \mathrm{~m}$, and it's spatial position can be solved by $14 x-14 y-5 z=1000$. Between the two interfaces, there is an ellipsoid $E$ the center of which is also the center of the model $(0.5 \mathrm{~km}, 0.5 \mathrm{~km},-0.5 \mathrm{~km})$. Its shape can be drawn out according to Ellipsoid parametric equation $\frac{(x-500)^{2}}{500^{2}}+\frac{(y-500)^{2}}{300^{2}}+\frac{(z+500)^{2}}{100^{2}}=1$

Longitudinal wave velocity $v_{p}$ and shear velocity $v_{s}$ of medium $I$ above the first interface are respectively 2 $\mathrm{km} / \mathrm{s}$ and $1.2 \mathrm{~km} / \mathrm{s}$. The seismic wave velocity distribution of medium II which exists between the two interfaces except the Ellipsoid is as follows:

$v_{p}(x, y, z)=2500+0.2(x-500)+0.1(y-500)-0.6(z+500)$

and

$v_{s}(x, y, z)=1500+0.1(x-500)+0.05(y-500)-0.3(z+500)$.

Below the second interface, medium III is uniform the velocity of which is $v_{p}=3.5 \mathrm{~km} / \mathrm{s}, v_{s}=2.1 \mathrm{~km} / \mathrm{s}$. The velocity of medium in the Ellipsoid is $v_{p}=3 \mathrm{~km} / \mathrm{s}, v_{s}=1.7 \mathrm{~km} / \mathrm{s}$. The coordinates of the source location are $56 \mathrm{~m}, 80 \mathrm{~m},-20 \mathrm{~m}$. For 3-D model, it can be subdivided into $100 \times 100 \times 100$ cubes in the interval of 10 meters in three directions.

The first-break travel time can be calculated by microgrid ray tracing on the $\mathrm{PC}$.

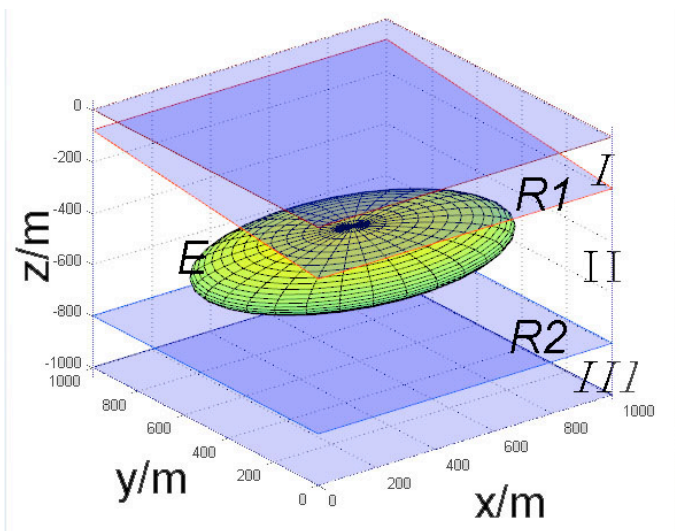

Fig. (2). 3D complex geological model.

\subsection{Forward Simulation}

Micro-grid ray path tracing algorithm is used in complex geological model to accomplish forward simulation. The ray trace path of seismic wave is the output. Then, the accurate first arrival travel time is calculated from the source point to each geophone, that is, the arrival time difference sequence of each geophone. Table $\mathbf{1}$ gives the location information of the source point and geophone array. Table 2 shows the arrival time difference sequences in different geophone arrays which are based on the first arrival travel time.

\subsection{BP-GA Inversion Algorithm in Condition of Random Noise}

When the geophone is working, it is disturbed by the noises. These noises are hard to be filtered clearly. Thus, there exists a time error. So the positioning error appears when there is a time error. Since the time error is random, if the simulation positioning disturbance is produced by random time disturbance and location error is estimated by simulation positioning disturbance, the location precision of all 
Table 1. Location information of the source point and geophone array (unit: meter).

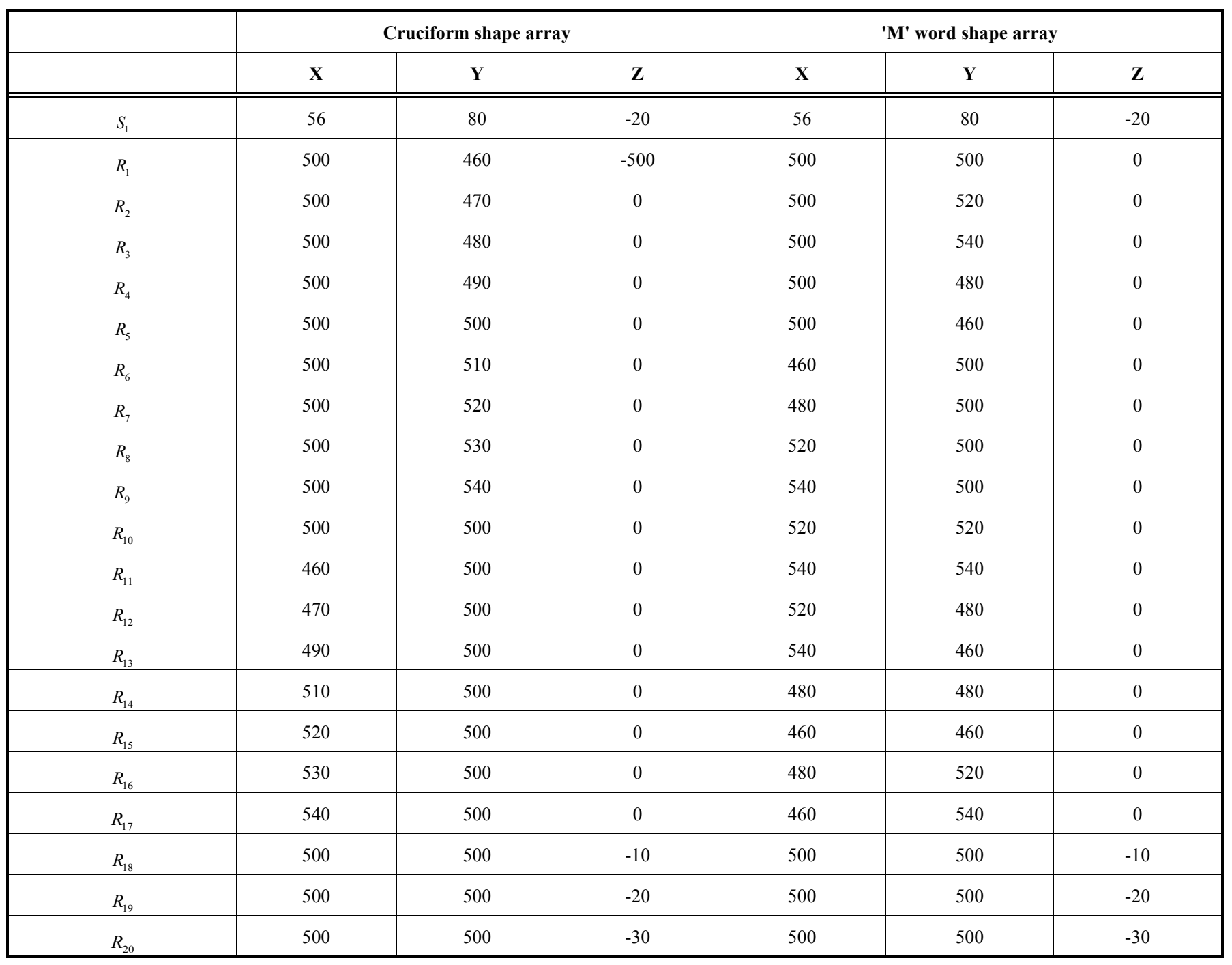

\begin{tabular}{|c|c|c|c|c|c|c|}
\hline & \multicolumn{3}{|c|}{ Square-like shape array } & \multicolumn{3}{c|}{ Octagonal shape array } \\
\hline & $\mathbf{X}$ & $\mathbf{Y}$ & $\mathbf{Z}$ & $\mathbf{X}$ & $\mathbf{Z}$ \\
\hline \hline$S_{1}$ & 56 & 80 & -20 & 56 & 80 \\
\hline$R_{1}$ & 500 & 460 & -500 & 500 & 500 & 520 \\
\hline$R_{2}$ & 500 & 470 & 0 & 500 & 540 \\
\hline$R_{3}$ & 500 & 480 & 0 & 500 & 480 \\
\hline$R_{4}$ & 500 & 490 & 0 & 500 & 0 \\
\hline$R_{5}$ & 500 & 500 & 0 & 500 & 460 \\
\hline$R_{6}$ & 500 & 510 & 0 & 460 & 500 \\
\hline$R_{7}$ & 500 & 520 & 0 & 480 & 500 \\
\hline$R_{8}$ & 500 & 530 & 0 & 520 & 0 \\
\hline$R_{9}$ & 500 & 540 & 0 & 540 & 500 \\
\hline$R_{11}$ & 500 & 500 & 0 & 520 & 500 \\
\hline
\end{tabular}


(Table 1) contd....

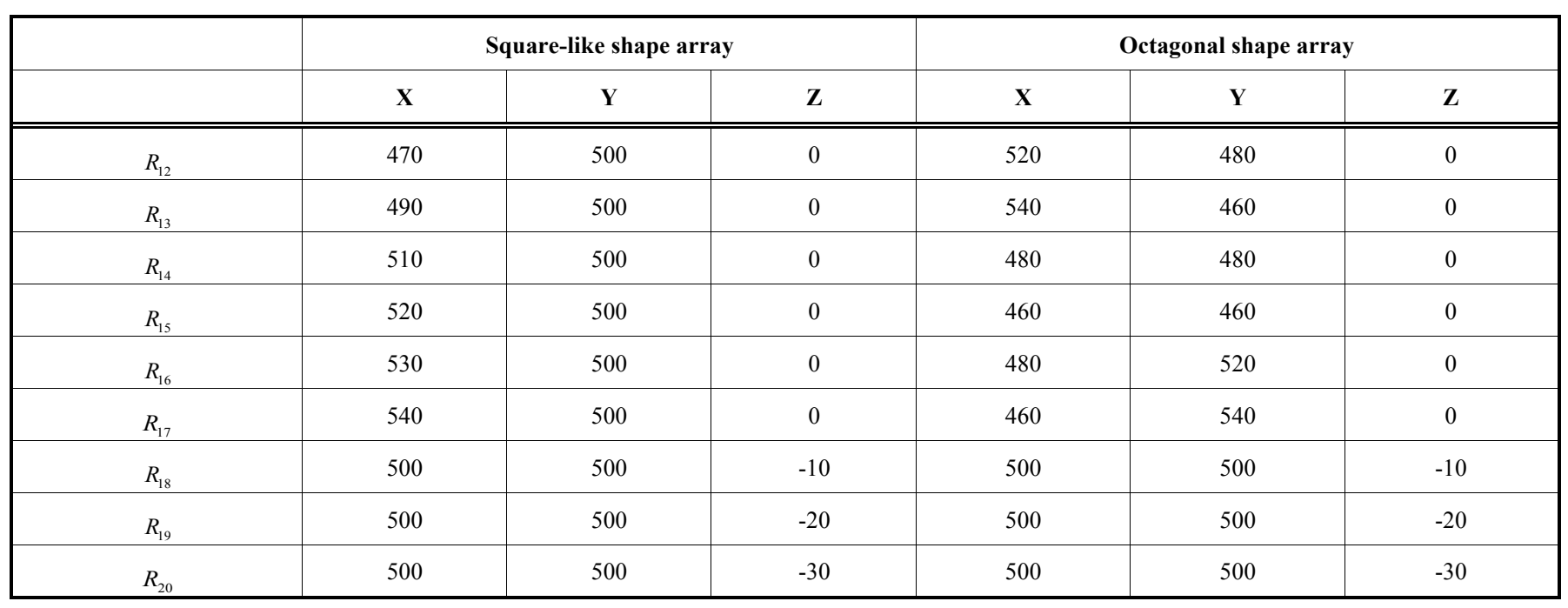

Table 2. Arrival time differences of 20 geophones in 4 kinds of arrays.

\begin{tabular}{|c|c|c|c|c|}
\hline$\Delta t_{1}$ & -0.0008 & -0.0009 & -0.0006 & -0.0007 \\
\hline$\Delta t_{3}$ & -0.0023 & -0.0025 & -0.0022 & -0.0021 \\
\hline$\Delta t_{4}$ & -0.0021 & -0.0022 & -0.0020 & -0.0024 \\
\hline$\Delta t_{6}$ & -0.0328 & -0.0325 & -0.0321 & -0.0318 \\
\hline$\Delta t_{7}$ & -0.0308 & -0.0309 & -0.0312 & -0.0311 \\
\hline$\Delta t_{8}$ & -0.0209 & -0.0210 & -0.0212 & -0.0211 \\
\hline$\Delta t_{12}$ & -0.0056 & -0.0057 & -0.0056 & -0.0056 \\
\hline$\Delta t_{13}$ & -0.0432 & -0.0433 & -0.0431 & -0.0431 \\
\hline$\Delta t_{14}$ & -0.0654 & -0.0653 & -0.0652 & -0.0651 \\
\hline$\Delta t_{15}$ & -0.0512 & -0.0511 & -0.0511 & -0.0510 \\
\hline$\Delta t_{16}$ & -0.0462 & -0.0463 & -0.0461 & -0.0462 \\
\hline$\Delta t_{17}$ & -0.0162 & -0.0163 & -0.0161 & -0.0162 \\
\hline$\Delta t_{18}$ & -0.0631 & -0.0632 & -0.0630 & -0.0632 \\
\hline
\end{tabular}

kinds of arrays can be compared. The normal distribution is used as the function of the simulation random noise distribution. The disturbance times should be as many as possible in simulation process. In this model test, after 150 disturbances, the error is no longer decreased. Therefore, random normal distribution is used to simulate the time disturbance whose standard deviation is $1 \mathrm{~ms}$. After 150 times, inversion is made under the condition that the time disturbance is added to the travel time. 
In the inversion, the objective function is a nonlinear regression model of travel time equation, that is

$$
T_{i}-T_{0}=\sqrt{\left(X_{i}-X_{0}\right)^{2}+\left(Y_{i}-Y_{0}\right)^{2}+\left(Z_{i}-Z_{0}\right)^{2}} / V_{p} \text {. }
$$

By micro-grid ray tracing method, the theoretical arrival time difference sequences are obtained of each geophone monitoring point. The sequences are made the learning sample, and genetic algorithm is completed and the BP neural network is optimized. After the optimizing, optimal weights and thresholds are obtained. BP neural network forecast is used within the limit of weights and threshold distribution. Then, the output is the wanted space coordinate of the source point. In BP algorithm, single invisible layer feed forward network is adopted while combining it with seismic source inversion model. The three-layer topologic structure includes the input layer, the hidden layer and the output layer. The number of input vector which represents the arrival time difference sequence is $n=20$, and the number of output vector which represents the coordinates of source point is $\mathrm{m}=3$ 。 Randomly, $\mathrm{N}$ groups of the weights and threshold between $[-1,1]$ are produced. According to Kolmogorov theorem, the number of hidden layer node is $2 n+1$. Therefore, the maximum hidden node is $\mathrm{Hmax}=41, \mathrm{Hmin}=3$. The neural network structure is $20-41-3$, and it has $20 \times 41+41$ $\times 3=943$ weights and $41+3=44$ thresholds. The result of BP network forecast is the wanted space coordinates of seismic source. The standard deviations of the true source location and inversion position which come from 4 geophone arrays are shown in Table 3 in which unit is meter.

Table 3. Standard deviation of inversion position and true location.

\begin{tabular}{|c|c|c|c|c|}
\hline & $\begin{array}{c}\text { Cruciform } \\
\text { shape array }\end{array}$ & $\begin{array}{c}\text { 'M' word } \\
\text { shape array }\end{array}$ & $\begin{array}{c}\text { Square-like } \\
\text { shape array }\end{array}$ & $\begin{array}{c}\text { Octagonal } \\
\text { shape array }\end{array}$ \\
\hline \hline $\mathrm{x}$ & 55.488 & 55.512 & 55.231 & 55.436 \\
\hline $\mathrm{y}$ & 78.543 & 78.332 & 78.752 & 78.224 \\
\hline $\mathrm{z}$ & -20.213 & -20.202 & -20.438 & -20.816 \\
\hline$\Delta \delta$ & 0.565 & 0.502 & 0.435 & 0.498 \\
\hline
\end{tabular}

Table 3 shows the predicting outcomes of BP-GA hybrid inversion algorithm including the inversion positioning coordinates $(\mathrm{x}, \mathrm{y}, \mathrm{z})$ and positioning error under constraint condition of the optimal weight and threshold parameter. From Table 3, it can be seen that when the network prediction error is in the range of $(0-0.6)$, it meets the demands of the seismic source inversion positioning error. When random disturbance is added, the inversion prediction result becomes reliable.

\subsection{Emulation Analysis of Geophone Arrays}

When the geophone array is in cruciform shape, M-word shape, square-like shape and octagonal shape, the source location error is shown as follows. This figure shows that for different positioning errors when the source point is in different depths the depth stepping is 100 meters.
Fig. (3) shows that the positioning error of cruciform shape array is less than 1 in the range of 0 to 400 meters, the positioning error of M-word shape array is less than 1 in the range of 0 to 700 meters, the positioning error of both Mword shape array and square-like shape array is less than 2 in the range of 700 to 1300 meters, the positioning error of square-like shape array is less than 1 in the range of 1300 to 1800 meters, the positing error of octagonal shape array is less than 1 in the range of 1800 to 2500 meters.

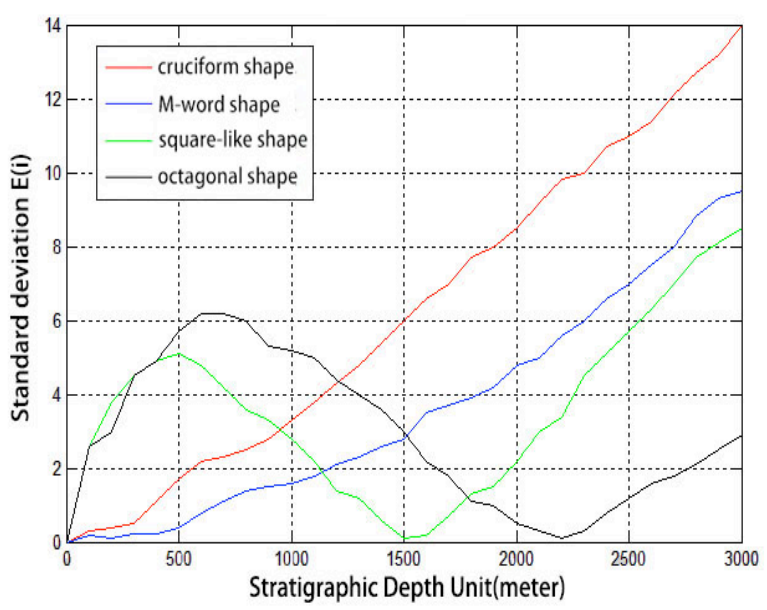

Fig. (3). Error map of source location.

\subsection{Analysis of the Relationships between Number of Geophone and Positioning Precision}

The changing curve of positing error when the number of geophone is added is shown in Fig. (4). The more the geophones are added, the less is the positioning error. However, when the number of geophones is increased to a certain number, the change in the curve of positioning error is slight. Fig. (4) represents the relationships between the number of geophones and the extent of change in positioning error. The number of geophones can be determined according to the positioning error.

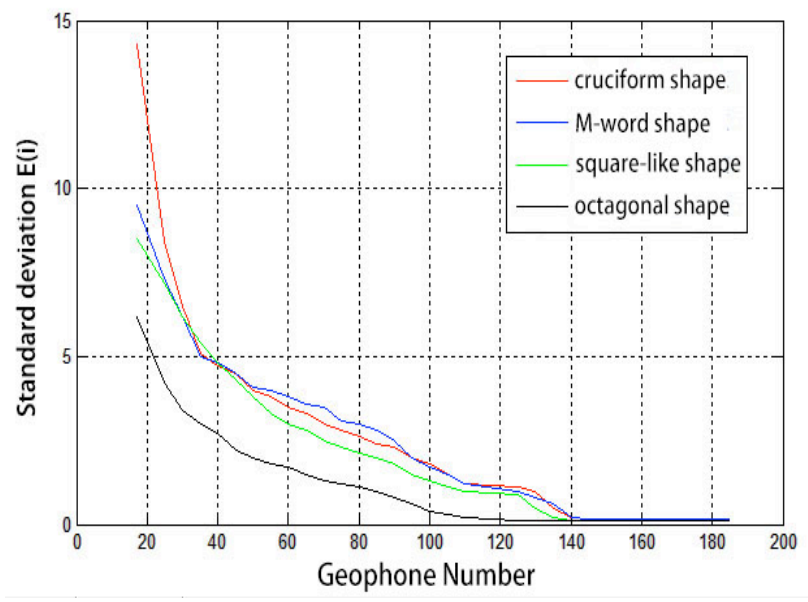

Fig. (4). Curves of change of positional error with geophone number.

The geophones are arranged in four kinds of schemes in simulation experiment. When the geophones are increased 
from 20 to 187, the change curves of positioning error are obtained with the increasing number of geophones. The vertical axis E(i) represents the error, and the horizontal axis represents the geophone number. As seen from the result of experiment, when geophones are arranged in cruciform shape and are added to 141 , the positional error curve tends to be smooth and the efficiency is in its highest level. When geophones are arranged in M-word shape and the number is added to 138 , the positional error curve tends to be smooth and the efficiency is in its highest level. When arranged in square-like shape and added to 135, the positional error curve tends to be smooth and efficiency is highest. When arranged in octagonal shape and added to 115 , the error curve tends to be smooth and the efficiency is also highest.

\section{CONCLUSION}

Micro-grid ray tracing method was used to establish the seismic geological model. Then, the forward simulation was carried out on every geophone array. When random disturbance was added, GA-BP method was used to retrieve the source location. Finally, through comparing the error of source location, the characteristics of four kinds of arrays were obtained, which were significant for the question as how to conduct array in seismic positioning and monitoring system. At the same time, according to the source positional error curves, when the geophone is in highest efficiency, the accurate, reasonable and efficient geophone array can be chosen. Results of positioning precision in four kinds of geophone array methods show that the algorithm presented in this paper is efficient and is of great significance in how to arrange geophones in seismic positioning and monitoring system in mining area.

\section{CONFLICT OF INTEREST}

The authors confirm that this article content has no conflict of interest.

\section{ACKNOWLEDGEMENTS}

The project was supported by the National Nature Science Foundation of China (Grant No. 61362020), Guangxi Natural Science Foundation (Grant No. 2012GX NSFBA053177, No. 2013GXNSFAA019327 and 2013GX
NSFFA019004), Guangxi Experiment Center of Information Science (No.20130112).

\section{REFERENCES}

[1] A. Adeyemi, and J. M. Harris, "Quasi-continuous reservoir monitoring with surface seismic data," Geophysical Prospecting, vol. 62, no. 1, pp. 117-132, 2014.

[2] Dmitry, I. Kitov, and L. Zerbo, "Perspectives of cross-correlation in seismic monitoring at the international data centre," Pure and Applied Geophysics, vol. 171, no. 3-5, pp. 439-468, 2014.

[3] C. Margherita, P. Roux, M. Campillo, and D. Dubucg, "Instantaneous phase variation for seismic velocity monitoring from ambient noise at the exploration scale," Geophysics, vol. 77, no. 4, pp. 3744, 2012.

[4] A. Kijko, and M. Sciocatti, "Optimal spatial distribution of seismic stations in mines," International Journal of Rock Mechanics and Mining Science and Geomechanics Abstracts, vol. 32, no. 6, pp. 607-615, 2012.

[5] J. Díaz, M. Ruíz, L. Crescentini, A. Amoruso, and J. Gallart, "Seismic monitoring of an Alpine mountain river," Journal of Geophysical Research: Solid Earth, vol. 119, no. 4, pp. 3276-3289, 2014.

[6] Fahrman, E. Westman, M. Karfakis, and K.D. Luxbacher, "Optimization of geophone array for monitoring geologic carbon sequestration using double-difference tomography," Journal of Energy Resources Technology-Transactions of the Asme, vol. 136, no. 1, 2014.

[7] F. Song, M.N. Toksöz, "Full-waveform based complete moment tensor inversion and source parameter estimation from down hole micro-seismic data for hydro fracture monitoring," Geophysics, vol. 40, no. 4, pp. 13-16, 2011.

[8] V. I. German, "Rock failure prediction in mines by seismic monitoring data," Journal of Mining Science, vol. 50, no. 2, pp. 288297, 2014.

[9] M. Krautblatter, and D. Draebing, "Pseudo 3-D P wave refraction seismic monitoring of permafrost in steep unstable bedrock," Journal of Geophysical Research: Earth Surface, vol. 119, no. 2, pp. 287-299, 2014.

[10] Nieto, F. Accaino, and L. Baradello, "Aquifer characterization and monitoring by active and passive seismic surveys," Procedia Engineering, vol. 89, pp. 1231-1234, 2014.

[11] C. Stotter, and A. Erika, "Evaluation of $3 \mathrm{C}$ microelectromechanical system data on a 2D line: direct comparison with conventional vertical-component geophone arrays and PS-wave analysis Geophysics," vol. 76, no. 3, pp. 79-87, 2011.

[12] O. Zakharova, S. Hainzl, and C. Bach, "Seismic moment ratio of aftershocks with respect to main shocks," Journal of Geophysical Research: Solid Earth, vol. 57, pp. 1319-1328, 2013.

[13] J. Wu, and J. Liu, "Calculation and application of mine microseismic location," Journal of Wuhan University of Science and Technology, vol. 36, no. 4, pp. 308-310, 2013.

Received: February 03, 2015

Revised: April 03, 2015

Accepted: May 25, 2015

(C) Zhang et al.; Licensee Bentham Open.

This is an open access article licensed under the terms of the (https://creativecommons.org/licenses/by/4.0/legalcode), which permits unrestricted, noncommercial use, distribution and reproduction in any medium, provided the work is properly cited. 